REVIEW

\title{
Does palliative care improve outcomes for patients with HIV/AIDS? A systematic review of the evidence
}

\author{
R Harding, D Karus, P Easterbrook, V H Raveis, I J Higginson, K Marconi
}

See end of article for authors' affiliations

.....................

Correspondence to: Dr Richard Harding, Department of Palliative Care and Policy, Guy's King's and St Thomas's School of Medicine, London SE5 9RS, UK

Richard.harding@kcl.ac.uk

Accepted for publication 13 May 2004
Sex Transm Infect 2005;81:5-14. doi: 10.1136/sti.2004.010132 necessary. Further studies are needed to both identify feasible methods and evaluate the apparent beneficial effect of palliative care on patient outcomes in the post-HAART era.
$\mathrm{T}$ he World Health Organization defines palliative care as "an approach that improves the quality of life of patients and their families facing the problems associated with life-threatening illness, through the prevention and relief of suffering by means of early identification and impeccable assessment and treatment of pain and other problems, physical, psychosocial and spiritual."

UNAIDS estimates at least 40 million people living with HIV/AIDS in 2003, and during 2003 there were three million HIV/AIDS related deaths, and five million new infections. ${ }^{2}$ The high prevalence of HIV related manageable symptoms including fatigue, anorexia, weight loss, depression, agitation and anxiety, nausea and vomiting, diarrhoea, cough, dyspnoea, fever, sweats, and pruritus, ${ }^{34}$ as well as often inadequate pain control $^{56}$ at all stages of HIV disease emphasise the need for palliative therapy.

Although widespread adoption of highly active antiretroviral therapy (HAART) in industrialised countries since 1996 has profoundly impacted on morbidity and mortality, the need for palliative care has continued. Firstly, HIV infected patients continue to die at a higher rate than the uninfected, and the virological failure rate with first and second line therapy remains high. ${ }^{8} 9$ Secondly, antiretroviral drugs are associated with a significant rate of toxicity and side effects ${ }^{10}$ such as peripheral neuropathy and gastrointestinal problems. Thirdly, as a result of survival prolongation, new comorbidities have become apparent such as end stage liver disease secondary to hepatitis $\mathrm{C}$ co-infection, myocardial infarction, ${ }^{11}$ cerebrovascular disease, ${ }^{12}$ and diagnoses associated with significant neurological and cognitive impairment such as progressive multifocal leucoencephalopathy. ${ }^{13}{ }^{14}$ Fourthly, some HIV related malignancies such as nonHodgkin's lymphoma, cervical carcinoma and colorectal and lung malignancies have not declined in incidence with HAART. ${ }^{15-17}$ Fifthly, a significant proportion of HIV infected individuals are unaware of their diagnosis, and present only with advanced disease.

The changing epidemiology of HIV disease in the era of HAART has resulted in new and evolving roles for palliative care, with a shift from the more conventional HIV palliative care of the 1980s and early 1990s to a greater focus on symptom control in patients who may continue to live for an extended period, or the need for active treatment for one HIV related condition and palliation for another simultaneously. ${ }^{18}$ However, in the developing world, where the majority of the HIV cases occur, and where antiretroviral drug availability remains limited, the principles of traditional palliative care still apply.

To date, there has been no comprehensive review of the different models of palliative care and their effectiveness for people affected by HIV/AIDS. Our objective was to systematically review the evidence base for the effectiveness of palliative care in improving patient outcomes in HIV/AIDS.

\section{METHODS}

\section{Search strategy}

In December 2003, we searched the following databases: Medline (1980-2003), CINAHL (1982-2003), PsychINFO (1980-2003), Embase (1980-2003), Applied Social Sciences Index and Abstracts (1987-2003), and Social Sciences

Abbreviations: $C C T$, community care team; HAART, highly active antiretroviral therapy; HCA, home care assistance; HST, home support team; ITDU, infectious and tropical diseases unit; PCU, palliative care unit; POS, Palliative Outcome Scale; QoL, quality of life; RCT,

randomised controlled trial; STAS, Support Team Assessment Schedule 


\begin{tabular}{l} 
Table 1 Grading criteria for studies \\
\hline Grade I (strong evidence) \\
RCTs or review of RCTs \\
- IA Calculation of sample size and accurate and standard \\
definition of appropriate outcome variables \\
- IB Accurate and standard definition of appropriate \\
outcome variables \\
IC Neither of the above \\
Grade II (fairly strong evidence) \\
Prospective study with a comparison group (non-randomised \\
controlled trial, good observational study or retrospective \\
study that controls effectively for confounding variables). \\
- IIA Calculation of sample size and accurate, standard \\
definition of appropriate outcome variables and \\
adjustment for the effects of important confounding \\
variables. \\
Grade One or more of the above \\
Retrospective or observational studies \\
- IIIA Comparison group, calculation of sample size, \\
accurate and standard definition of appropriate outcome \\
variables. \\
IIIB Two or more of the above \\
IIIC One or none of the above* \\
Grade IV (weak evidence) \\
Cross sectional study, Delphi exercise, consensus of experts \\
\hline * Modified from Clinical Guidance Outcomes Group. \\
* ${ }^{20}$
\end{tabular}

Citation Index (1981-2003). The search terms were (palliative/hospice*/terminal care/supportive care/terminally ill/palliat*/dying/end of life/home care/advanced/comfort care) and (HIV/AIDS). In order to obtain as comprehensive a search as possible, ${ }^{19}$ terms were selected to identify the range of models commonly used as proxies for palliative care, or care mixes that include palliation as a component.

The criteria for inclusion of studies were original research paper in English language reporting on human subjects; a minimum of $50 \%$ of study population infected with HIV (or HIV infected subsample analysed and reported separately); the provision of a basic service description and/or evaluation of patient outcomes or patient satisfaction with care. Studies that incorporated elements of palliative care (that is, pain and symptom control, psychosocial support, terminal care) were included regardless of whether they explicitly used the term.

\section{Analysis}

Data items were extracted using a standard proforma: year of publication, setting/country, HIV disease stage and referral criteria to service, service description and palliative approach, sample size, study design, outcomes, and findings.

The studies were independently graded by two researchers (RH and DK) using a well described hierarchy of evidence ${ }^{20}$ (table 1). Studies were further grouped according to intervention model used. A formal meta-analysis with statistical pooling of results across studies was not possible because of the absence of both appropriate study designs and standardised outcome measures.

\section{RESULTS}

\section{Characteristics of eligible studies}

The 32 eligible studies originated from the following countries: United Kingdom $(n=10)$; United States $(n=9)$; sub-Saharan Africa $(\mathrm{n}=5)$; Canada $(\mathrm{n}=2)$; Australia $(\mathrm{n}=1)$; France $(\mathrm{n}=1)$; Italy $(\mathrm{n}=2)$; Netherlands $(\mathrm{n}=1)$; and Norway $(\mathrm{n}=1)$ (table 2 ). Of those countries that adopted widespread use of HAART from 1996-7 (that is, excluding the five studies based in sub-Saharan Africa), 18 studies were published pre-HAART and nine post-HAART.

Thirty four different palliative care services were described in 32 different studies, although only about half of these services (18/34) stated explicitly that their patient management was palliative. Of the 34 services, 22 had been evaluated in 17 studies. The different service models were home based care $(n=15)$; home palliative care/hospice at home $(n=7)$; hospice inpatient $(n=4)$; hospital inpatient palliative care $(n=4)$; specialist AIDS inpatient unit $(n=2)$; and hospital inpatient and outpatient care $(n=2)$ (table 2$)$.

In general, these services offered various combinations of usual disciplines found in palliative care, including consultants in palliative medicine, palliative nurse specialists, pain and symptom control, on-call home care, education and advice, counselling, psychosocial care, pastoral/spiritual care, occupational therapy, social work, dietetics, a focus on family and patient support, and bereavement counselling.

Patient eligibility for the services was described for 30/34 services and ranged from any stage of HIV disease $(n=12)$ to only those with an AIDS diagnosis $(n=11)$, or advanced/ terminal AIDS $(n=7)$. As would be expected, the services that offered care to all HIV infected patients were less likely to name palliation as part of the care package $(2 / 12)$ than were services specifically for people with an AIDS diagnosis (7/11); or those for advanced disease services (6/7). The proportion of services that incorporated an evaluation of their service varied with the type of service model: home based care $(n=13 / 15)$; home palliative care and hospice at home $(\mathrm{n}=5 / 7)$; hospital palliative care $(\mathrm{n}=2 / 4)$; hospital inpatient and outpatient care $(n=1 / 2)$; hospice inpatient $(n=1 / 4)$; specialist AIDS inpatient unit $=(0 / 2)$.

The clinical outcomes used in the evaluation of palliative care services varied. Four studies used the Support Team Assessment Schedule (STAS) ${ }^{21}$ which was designed to assess domains including pain and symptom control, patient and family anxiety, patient and family insight, and communication. Other studies assessed satisfaction with care, medical procedures and investigations, perceived quality of care, patient involvement, support, and quality of life (generally with non-standardised measures).

Of the 22 services that had been evaluated in 17 studies, the strength of the evidence in each study was graded as grade $1(n=1)$; grade $2(n=2)$; grade $3(n=7)$; grade 4 $(\mathrm{n}=1)$. Six studies used only qualitative data.

\section{Findings by evidence grade}

Grade $1(n=1)$ : strong evidence

In a randomised controlled trial (RCT), integrated multiprofessional case managed home care $(n=29)$ showed a possible advantage over standard home care $(n=28)$ in terms of both quality of wellbeing and survival (magnitude unreported, figurative representation of data only). However, this did not reach statistical significance. ${ }^{22}$

\section{Grade $2(n=2)$ : fairly strong evidence}

In an observational study 10 patients accessing home palliative care in addition to hospital care were compared to 32 who were attending hospital care only. Small sample size, death and attrition disallowed statistical analysis, and no difference in quality of wellbeing was reported although number of hospital admissions and mean number of days for each admission and overall costs were lower for those receiving home care. In a further observational study, relative to 77 patients receiving standard hospital inpatient care, 10 patients attending a hospital inpatient palliative care unit (PCU) were less likely to have been prescribed antiretrovirals $(34 \% \vee 10 \%)$ and antimicrobial agents $(60 \% \vee 0 \%)$, more likely to have ceased treatment for curable infections $(23 \% \mathrm{v}$ $80 \%$ ), and more likely to have been prescribed step 3 analgesics $(18 \% \vee 80 \%){ }^{23}$ Depression was also far less common in the PCU (55\% $v 10 \%)$, although a similar frequency of pain was assessed (46\% v 50\%). However, 
Table 2 Service descriptions and evaluation evidence

\begin{tabular}{|c|c|c|c|c|c|}
\hline Ref/grade & $\begin{array}{l}\text { Setting and country/ } \\
\text { disease stage } \\
\text { eligibility criteria }\end{array}$ & Service description & Design and sample size & Outcomes & Findings \\
\hline \multicolumn{6}{|c|}{ Hospice at home/home palliative care } \\
\hline $\begin{array}{l}\text { Tramarin } \\
1992^{71} / \text { IIB }\end{array}$ & $\begin{array}{l}\text { Home palliative } \\
\text { care assistance, } \\
\text { Vicenza Italy/ } \\
\text { terminal AIDS or } \\
\text { heavy motor or } \\
\text { vision deficit }\end{array}$ & $\begin{array}{l}\text { Multidisciplinary home } \\
\text { care assistance (HCA) } \\
\text { consisting of nurses, } \\
\text { family doctor, psychologist, } \\
\text { volunteers, social workers } \\
\text { and infectious disease } \\
\text { specialists. Aimed to } \\
\text { improve quality of life } \\
\text { through principles of } \\
\text { palliative care and limiting } \\
\text { inpatient service use }\end{array}$ & $\begin{array}{l}6 \text { month prospective } \\
\text { comparison of } n=10 \text { patients } \\
\text { accessing HCA and hospital } \\
\text { care to } n=32 \text { with similar } \\
\text { disease staging accessing only } \\
\text { hospital care }\end{array}$ & $\begin{array}{l}\text { Quality of wellbeing } \\
\text { scale calculated } \\
\text { weekly }\end{array}$ & $\begin{array}{l}\text { Quality of wellbeing data not } \\
\text { presented in numeric form but } \\
\text { graph suggests no significant } \\
\text { difference. Fewer hospital } \\
\text { inpatient days reported for those } \\
\text { accessing home care ( } n=7,127 \\
\text { days) than comparison group } \\
\text { (n=35, } 866 \text { days) and costs } \\
\text { lower for those with advanced } \\
\text { disease accessing home care } \\
(\$ 17237 v \$ 27764)\end{array}$ \\
\hline $\begin{array}{l}\text { Koffman } \\
1996^{25} / \text { IIIC }\end{array}$ & $\begin{array}{l}\text { Home based } \\
\text { hospice, London } \\
\text { UK/advanced } \\
\text { HIV/AIDS }\end{array}$ & $\begin{array}{l}24 \text { hour palliative nursing, } \\
\text { night sitting, consultants in } \\
\text { palliative medicine }\end{array}$ & $\begin{array}{l}\text { Single group longitudinal; } \\
\text { from entry to last week of } \\
\text { life/discharge. } N=36\end{array}$ & $\begin{array}{l}\text { STAS quality of life } \\
\text { (QoL): pain and } \\
\text { symptom control, } \\
\text { patient and family } \\
\text { anxiety, patient and } \\
\text { family insight, } \\
\text { patient/family/ } \\
\text { professional } \\
\text { communication }\end{array}$ & $\begin{array}{l}\text { All items showed trend to } \\
\text { improvement, with two reaching } \\
\text { statistical significance: symptom } \\
\text { control (from } 6 \% \text { to } 33 \% \\
\text { reporting it as no problem, } \\
p=0.0009 \text { ) and family insight } \\
\text { (from } 33 \% \text { to } 67 \% \text { reporting as } \\
\text { no problem } p=0.0006 \text { ) }\end{array}$ \\
\hline $\begin{array}{l}\text { Butters } \\
1995^{28} / \text { IIIC }\end{array}$ & $\begin{array}{l}2 \text { hospital and home } \\
\text { care, London UK/ } \\
\text { HIV/AIDS ( } 85 \% \\
\text { diagnosed AIDS) }\end{array}$ & $\begin{array}{l}\text { Multidisciplinary advice } \\
\text { and support in hospital } \\
\text { and at home. (A) Home } \\
\text { support team (HST)- } \\
\text { nursing staff, general } \\
\text { practitioner, welfare rights } \\
\text { adviser and occupational } \\
\text { therapist. Early intervention } \\
\text { inc. asymptomatic HIV. (B) } \\
\text { Community care team } \\
\text { (CCT) - consultant, nurse } \\
\text { specialists, social worker, } \\
\text { dietician, occupational } \\
\text { therapist, registrar. } \\
\text { Late/end stage care }\end{array}$ & $\begin{array}{l}\text { Prospective, multicentre } \\
\text { longitudinal from referral to } \\
\text { death/discharge. } N=234\end{array}$ & $\begin{array}{l}\text { STAS: severity and } \\
\text { nature of patient } \\
\text { problems (pain and } \\
\text { symptom control, } \\
\text { anxiety) }\end{array}$ & $\begin{array}{l}\text { Significant changes from } \\
\text { referral to } 6 \text { weeks before } \\
\text { death. (A) Worsening symptom } \\
\text { control (from } 22 \% \text { to } 46 \% \text { rated } \\
\text { severe, } p=0.04)) \text {. (B) Improving } \\
\text { predictability }(p=0.000002) \text {, } \\
\text { spiritual ( } p=0.005) \text {, patient/ } \\
\text { family communication } \\
(p=0.02) \text {, patient insight } \\
(p=0.04)\end{array}$ \\
\hline \multirow[t]{2}{*}{$\begin{array}{l}\text { Butters } \\
1992^{26} / \text { IIIC }\end{array}$} & $\begin{array}{l}2 \text { community } \\
\text { teams, London UK/ } \\
\text { HIV/AIDS (84\% } \\
\text { diagnosed AIDS) }\end{array}$ & $\begin{array}{l}\text { Multidisciplinary palliative } \\
\text { family and patient } \\
\text { support: symptom control, } \\
\text { counselling, bereavement } \\
\text { follow up, education and } \\
\text { advice, } 24 \text { hour on call } \\
\text { nursing and terminal care, } \\
\text { clinical liaison. Coordination } \\
\text { and support rather than care } \\
\text { management }\end{array}$ & $\begin{array}{l}2 \text { centre longitudinal, } \\
\text { entry to last week of life/ } \\
\text { discharge. } N=140 \\
\text { e }\end{array}$ & $\begin{array}{l}\text { STAS QoL: pain } \\
\text { and symptom } \\
\text { control, anxiety, } \\
\text { practical aid }\end{array}$ & $\begin{array}{l}\text { Significant improvements after } \\
2 \text { weeks from referral: pain } \\
\text { (from } 51 \% \text { at referral to } 66 \% \\
\text { after } 2 \text { weeks rated no problem, } \\
p=0.01 \text { ), symptom control ( } 4 \% \\
\text { to } 16 \% p=0.05) \text { and patient } \\
\text { anxiety }(7 \% \text { to } 16 \% p=0.05)\end{array}$ \\
\hline & & & & & $\begin{array}{l}\text { Significant changes from } \\
\text { referral to last week before } \\
\text { death: Pain no problem } 51 \% \text { to } \\
66 \% p=0.0001 \text {, symptom } \\
\text { control } 4 \% \text { to } 24 \% p=0.01 \text {, } \\
\text { anxiety } 7 \% \text { to } 47 \% p=0.00005 \text {. } \\
\text { However, } 33 \% \text { reported } \\
\text { symptoms as severe in last week } \\
\text { of care }\end{array}$ \\
\hline $\begin{array}{l}\text { Kimball } \\
1996^{29} / \text { IIIC }\end{array}$ & $\begin{array}{l}\text { Home hospice care } \\
\text { USA/AIDS }\end{array}$ & No service description & $\begin{array}{l}\text { Retrospective cohort chart } \\
\text { review, last } 2 \text { weeks of life. } \\
N=185\end{array}$ & $\begin{array}{l}\text { Pharmacological } \\
\text { management of } \\
\text { pain and } \\
\text { discomfort }\end{array}$ & $\begin{array}{l}\text { Those reporting pain and } \\
\text { discomfort rose from } 54 \% \text { early } \\
\text { in } 2 \text { weeks before death to } 68 \% \\
\text { in final } 48 \text { hours. Over } 2 \text { weeks } \\
88 \% \text { received opioid analgesic, } \\
\text { with } 62 \% \text { of these experiencing } \\
\text { relief thereafter }\end{array}$ \\
\hline $\begin{array}{l}\text { Butters } \\
1993^{24} / \text { IIIC }\end{array}$ & $\begin{array}{l}\text { Community support } \\
\text { team, London } \\
\text { UK/late stage HIV/ } \\
\text { AIDS illness/ } \\
\text { uncontrolled pain/ } \\
\text { symptoms, or need } \\
\text { psychological, } \\
\text { spiritual or home } \\
\text { support }\end{array}$ & $\begin{array}{l}\text { Multidisciplinary team (2 } \\
\text { doctors, } 3 \text { clinical nurse } \\
\text { specialists, dietician, } \\
\text { occupational therapist) to } \\
\text { increase choice of place } \\
\text { for care/death. Symptom } \\
\text { control, patient, and carer } \\
\text { counselling, } 24 \text { hour on call, } \\
\text { education, and advice on } \\
\text { diagnosis, nursing, and } \\
\text { terminal care. Coordination } \\
\text { and support of hospital and } \\
\text { home care, rather than } \\
\text { assume responsibility for } \\
\text { care }\end{array}$ & $\begin{array}{l}\text { Single group longitudinal, } \\
3-4 \text { weeks from referral } \\
(\mathrm{N}=19 / 125 \text { eligible }) \text { and } \\
4-6 \text { weeks later }(\mathrm{N}=6) \\
\end{array}$ & $\begin{array}{l}9 \text { STAS items and } \\
\text { satisfaction in final } \\
\text { weeks of life. Score } \\
\text { range from } 0=\text { no } \\
\text { problem to } 4= \\
\text { severe problems }\end{array}$ & $\begin{array}{l}\text { Care rated as good/excellent by } \\
\text { all. Communication with clinical } \\
\text { staff outside the team rated as } \\
\text { poor. } 2-4 \text { weeks from entering } \\
\text { care mean scores above } 2 \text { with } \\
\text { symptom control, pain control } \\
\text { and patient anxiety. } 18 / 19 \\
\text { reported problems with } \\
\text { symptom control. Few patients } \\
\text { reported severe ratings in final } \\
\text { weeks: none for pain, } 3 \text { for } \\
\text { symptom control, one for patient } \\
\text { anxiety. Favourable comments } \\
\text { on the role of "talking" care } \\
(n=18 / 19) \text { and with patients' } \\
\text { control over care }(n=15 / 19) \text {. }\end{array}$ \\
\hline
\end{tabular}


Table 2 Continued

\begin{tabular}{|c|c|c|c|c|c|}
\hline Ref/grade & $\begin{array}{l}\text { Setting and country/ } \\
\text { disease stage } \\
\text { eligibility criteria }\end{array}$ & Service description & Design and sample size & Outcomes & Findings \\
\hline Crowe $2001^{72} /-$ & $\begin{array}{l}\text { Home based } \\
\text { hospice, South } \\
\text { Africa/none } \\
\text { specified }\end{array}$ & $\begin{array}{l}\text { Nurses and trained rural } \\
\text { community carers, home } \\
\text { visiting, physical and } \\
\text { medical care, counselling, } \\
\text { and emotional/spiritual } \\
\text { support, social workers. } \\
\text { In-patient care for those } \\
\text { with greatest need. } \\
\text { Orphan support }\end{array}$ & No evaluation & & \\
\hline Martin $1988^{73} /-$ & $\begin{array}{l}\text { Home Care and } \\
\text { Hospice program, } \\
\text { San Francisco } \\
\text { USA/none } \\
\text { specified }\end{array}$ & $\begin{array}{l}\text { Team supports both home } \\
\text { care and } 15 \text { bed } \\
\text { residential care, mainly } \\
\text { HIV/AIDS. Physician } \\
\text { consultant advises } \\
\text { patients' primary } \\
\text { physician }\end{array}$ & No evaluation & & \\
\hline Martin $1986^{74} /-$ & $\begin{array}{l}\text { Home care and } \\
\text { hospice program, } \\
\text { San Francisco } \\
\text { USA/AIDS }\end{array}$ & $\begin{array}{l}\text { Multidisciplinary: physical } \\
\text { and occupational } \\
\text { therapists, attendants } \\
\text { personal care, daily I } \\
\text { iving activities), nurses } \\
\text { (symptom control) social } \\
\text { workers (psychosocial } \\
\text { care), volunteers (practical } \\
\text { support), rehabilitation } \\
\text { therapists (reducing } \\
\text { discomfort, improving } \\
\text { environment), consultants } \\
\text { (care planning, advocacy, } \\
\text { and education) }\end{array}$ & No evaluation & & \\
\hline \multicolumn{6}{|l|}{ Inpatient hospice } \\
\hline $\begin{array}{l}\text { Gibbs } \\
1997^{27} / \mathrm{IIIC}\end{array}$ & $\begin{array}{l}\text { Generic hospice, } \\
\text { London UK/ } \\
\text { advanced HIV } \\
\text { disease }\end{array}$ & $\begin{array}{l}\text { Terminal care. No service } \\
\text { description }\end{array}$ & $\begin{array}{l}\text { Retrospective } \\
\text { longitudinal. } N=26\end{array}$ & Symptom control & $\begin{array}{l}\text { Weakness (77\%), immobility } \\
\text { ( } 73 \% \text { ), and weight loss ( } 62 \% \text { ) } \\
\text { most severe symptoms at } \\
\text { admission, did not improve. } \\
\text { Severe pain for } 31 \% \text { at } \\
\text { admission, reduced to mild/ } \\
\text { none for all but } 1 \text { patient. } \\
\text { Significant improvement of other } \\
\text { symptoms (nausea, vomiting, } \\
\text { diarrhoea, constipation, } \\
\text { dyspnoea, confusion: not } \\
\text { quantified). No symptoms } \\
\text { initially rated absent or mild } \\
\text { worsened during stay }\end{array}$ \\
\hline Murie $1992^{75} /-$ & $\begin{array}{l}\text { AIDS hospice, } \\
\text { Edinburgh UK/ } \\
\text { AIDS: priority } \\
\text { given to terminal } \\
\text { patients }\end{array}$ & $\begin{array}{l}\text { Respite, convalescence, } \\
\text { Nurses and trained rural } \\
\text { community carand terminal } \\
\text { care. Medical nursing and } \\
\text { paramedical staff. } 12 \text { single } \\
\text { plus } 4 \text { double rooms. } \\
\text { Emphasis on liaison with } \\
\text { community drug health and } \\
\text { psychology services. } \\
\text { Emotional support, art, and } \\
\text { expressive classes, } \\
\text { complementary therapy. } \\
\text { Family care and inclusion }\end{array}$ & No evaluation & & \\
\hline Ley $1988^{76} /-$ & $\begin{array}{l}\text { AIDS hospice, } \\
\text { Toronto Canada/ } \\
\text { terminal AIDS }\end{array}$ & $\begin{array}{l}\text { Terminal care } 13 \text { beds } \\
\text { (including } 1 \text { respite bed). } \\
\text { Complementary therapies, } \\
\text { clinical staff, social worker, } \\
\text { psychosocial, and spiritual } \\
\text { care. Palliative approach } \\
\text { seen as more active than for } \\
\text { traditional cancer care. } \\
\text { Counselling for family/ } \\
\text { carers/friends/patients. } \\
\text { Outreach support for those } \\
\text { waiting for admission }\end{array}$ & No evaluation & & \\
\hline
\end{tabular}


Table 2 Continued

\begin{tabular}{|c|c|c|c|c|c|}
\hline Ref/grade & $\begin{array}{l}\text { Setting and country/ } \\
\text { disease stage } \\
\text { eligibility criteria }\end{array}$ & Service description & Design and sample size & Outcomes & Findings \\
\hline Moss $1988^{77} /-$ & $\begin{array}{l}\text { AIDS inpatient } \\
\text { hospice, London } \\
\text { UK/AIDS }\end{array}$ & $\begin{array}{l}\text { Rehabilitation, } \\
\text { convalescence, terminal } \\
\text { care. Patient centred } \\
\text { multidisciplinary holistic } \\
\text { care. All care in consultation } \\
\text { with patient, aims to improve } \\
\text { QoL through symptom } \\
\text { control and active treatment } \\
\text { where appropriate. Spiritual } \\
\text { care and counselling. } 9 \\
\text { beds, plus home support. } \\
\text { Physiotherapy and } \\
\text { occupational therapy; no } \\
\text { diagnostic facilities or } \\
\text { resuscitation. CNS, nurses, } \\
\text { doctors, counsellor, social } \\
\text { worker, chaplain, } \\
\text { housekeeper. Family facilities }\end{array}$ & No evaluation & & \\
\hline \multicolumn{6}{|c|}{ Hospital palliative care } \\
\hline $\begin{array}{l}\text { Vincent } \\
2000^{23} / I I B\end{array}$ & $\begin{array}{l}\text { Two models: (A) } \\
\text { Advanced AIDS } \\
\text { care in hospital } \\
\text { Infectious and } \\
\text { tropical disease } \\
\text { unit. (B) Hospital } \\
\text { palliative care unit, } \\
\text { Paris and Villejuif } \\
\text { France/(A) } \\
\text { Biomedical } \\
\text { admissions. (B) } \\
\text { "Qualitative" } \\
\text { admission criteria in } \\
\text { PCU (eg, suffering of } \\
\text { patient/family or } \\
\text { carer exhaustion) }\end{array}$ & $\begin{array}{l}\text { Care not described, } \\
\text { although (A) "Not } \\
\text { devoted to palliative } \\
\text { care" and (B) "Primary } \\
\text { objective of palliation" }\end{array}$ & $\begin{array}{l}\text { Prospective multidisciplinary } \\
\text { comparing methods of care } \\
\text { between ITDU ( } N=77 \text { ) and } \\
\text { PCU ( } N=10) \text {. Symptom self } \\
\text { assessment for only } 52 / 68 \\
\text { patients because of patient } \\
\text { inability/unwillingness }\end{array}$ & $\begin{array}{l}\text { Drugs administered, } \\
\text { procedures, and } \\
\text { investigations } \\
\text { symptoms assessed } \\
\text { weekly. Comparison } \\
\text { of different } \\
\text { therapeutic } \\
\text { objectives }\end{array}$ & $\begin{array}{l}\text { Sample sizes too different for } \\
\text { statistical analysis. ITDU } v \text { PCU } \\
\text { per hospitalisation: } \\
\text { antiretrovirals prescribed in } 34 \% \\
v 10 \% \text {; treatment of curable } \\
\text { infections ceased in } 23 \% \text { v } 80 \% \text {; } \\
\text { antimicrobial agents in } 60 \% v \\
0 \% \text {; Step } 2 \text { and step } 3 \\
\text { analgesics in } 57 \% \text { and } 18 \% v \\
20 \% \text { and } 80 \% \text {; Similar } \\
\text { frequency of pain ( } 46 \% \text { and } \\
48 \% \text { ) depression assessed in } \\
55 \% \text { of ITDU and } 7 \% \text { in PCU }\end{array}$ \\
\hline $\begin{array}{l}\text { Lucas } \\
1997^{30} / \mathrm{IIIC}\end{array}$ & $\begin{array}{l}\text { Inpatient palliative } \\
\text { care unit, New } \\
\text { South Wales } \\
\text { Australia/AIDS }\end{array}$ & $\begin{array}{l}8 \text { AIDS designated beds } \\
\text { in } 35 \text { bed palliative care } \\
\text { unit in public hospital. } \\
\text { Twice weekly visits by } \\
\text { pharmacist specialising } \\
\text { in AIDS palliative care: } \\
\text { assesses appropriateness } \\
\text { of prescribing, advises } \\
\text { on adverse reactions, } \\
\text { interactions, } \\
\text { contraindications and } \\
\text { therapeutic alternatives, } \\
\text { provides information to staff } \\
\text { and patients, applies for } \\
\text { restricted drugs, updates, } \\
\text { case notes. }\end{array}$ & $\begin{array}{l}\text { Retrospective rating of weekly } \\
\text { consultations with specialist } \\
\text { palliative AIDS pharmacist } \\
\text { advice over } 6 \text { months. Impact } \\
\text { independently evaluated on } 6 \\
\text { point scale by three clinicians. } \\
\mathrm{N}=11\end{array}$ & $\begin{array}{l}\text { Clinical significance } \\
\text { of advice given by } \\
\text { palliative AIDS } \\
\text { pharmacist }\end{array}$ & $\begin{array}{l}\text { Not quantified. High compliance } \\
\text { with recommendations. Most } \\
\text { common interventions: } \\
\text { rationalisation of inappropriate } \\
\text { regimens; warnings about drug } \\
\text { interactions. General } \\
\text { conclusion: among AIDS } \\
\text { patients, palliative care is } \\
\text { enhanced with availability of } \\
\text { pharmacist with specialist } \\
\text { knowledge of AIDS therapeutics. }\end{array}$ \\
\hline $\begin{array}{l}\text { Cumming } \\
1993^{78} /-\end{array}$ & $\begin{array}{l}\text { Non-HIV hospital } \\
\text { palliative care unit, } \\
\text { Vancouver Canada/ } \\
\text { prognosis }<1 \text { year, } \\
2 / 3 \text { AIDS }\end{array}$ & $\begin{array}{l}\text { Respite beds in generic } \\
\text { hospital palliative care } \\
\text { unit. Goals of pain and } \\
\text { symptom reassessment, } \\
\text { investigation, care for } \\
\text { caregiver }\end{array}$ & No evaluation & & \\
\hline $\begin{array}{l}\text { Singh } \\
1991^{79} /-\end{array}$ & $\begin{array}{l}\text { Dedicated hospital } \\
\text { ward, London UK/ } \\
\text { symptomatic HIV/ } \\
\text { AIDS }\end{array}$ & $\begin{array}{l}\text { Dedicated } 17 \text { bed hospital } \\
\text { ward for HIV/AIDS. } \\
\text { Provides both continuing } \\
\text { active treatment ( } 34 \% \text { ) } \\
\text { and no active treatment } \\
(66 \%)\end{array}$ & No evaluation & & \\
\hline \multicolumn{6}{|l|}{ Home based care } \\
\hline $\begin{array}{l}\text { Nickel } \\
1996^{22} / 1 \mathrm{~B}\end{array}$ & $\begin{array}{l}\text { Home care case } \\
\text { management, Ohio, } \\
\text { USA/AIDS }\end{array}$ & $\begin{array}{l}\text { Care monitoring and } \\
\text { direction by } \\
\text { comprehensive assessment } \\
\text { and care planning and } \\
\text { review by multidisciplinary } \\
\text { team: nursing staff, doctors, } \\
\text { social worker, psychiatrist, } \\
\text { nutritionist, clergy, } \\
\text { pharmacist, and dentist. } \\
\text { Services available on call }\end{array}$ & $\begin{array}{l}\text { Randomised controlled } \\
\text { trial (RCT). } N=57\end{array}$ & $\begin{array}{l}\text { Quality of Well } \\
\text { Being Scale, } \\
\text { Physical Self } \\
\text { Maintenance Scale, } \\
\text { Functional Activities } \\
\text { Questionnaire }\end{array}$ & $\begin{array}{l}\text { Case managed group showed } \\
\text { observable advantage in quality } \\
\text { of wellbeing and survival (not } \\
\text { quantified), but did not reach } \\
\text { statistical significance }\end{array}$ \\
\hline
\end{tabular}


Table 2 Continued

\begin{tabular}{|c|c|c|c|c|c|}
\hline Ref/grade & $\begin{array}{l}\text { Setting and country/ } \\
\text { disease stage } \\
\text { eligibility criteria }\end{array}$ & Service description & Design and sample size & Outcomes & Findings \\
\hline Foley $1995^{31}$ /IV & $\begin{array}{l}\text { Home care, New } \\
\text { York USA/ } \\
\text { symptomatic HIV/ } \\
\text { AIDS }\end{array}$ & $\begin{array}{l}\text { Enhance QoL, providing } \\
\text { home nursing and case } \\
\text { management, dietetics, } \\
\text { rehabilitation, equipment, } \\
\text { social work, respite, mental } \\
\text { health services, laboratory } \\
\text { and pharmacy services }\end{array}$ & Cross sectional. $\mathrm{N}=52$ & $\begin{array}{l}\text { Patient satisfaction } \\
\text { (excellent }=1 \\
\text { poor }=5 \text { ) }\end{array}$ & $\begin{array}{l}\text { Rated quality of nursing care as } \\
\text { excellent/very good (mean } 1.6 \text { ), } \\
\text { thought had more control over } \\
\text { hospital admissions (mean } 2.2 \text { ), } \\
\text { vast majority (67\%) reported } \\
\text { their health status remained } \\
\text { unchanged following admission } \\
\text { to the service. High value and } \\
\text { satisfaction placed on contact } \\
\text { and communication (81\%) }\end{array}$ \\
\hline $\begin{array}{l}\text { McCann } 1991^{36} / \\
\text { qualitative }\end{array}$ & $\begin{array}{l}\text { Home support team, } \\
\text { London, UK/HIV/ } \\
\text { AIDS }\end{array}$ & $\begin{array}{l}\text { Hospital based specialist } \\
\text { home support team, } \\
\text { parallels cancer support, to } \\
\text { allow home care and to } \\
\text { release hospital beds for } \\
\text { acute care. Coordinate and } \\
\text { provide continuous care } \\
\text { between hospital and } \\
\text { community teams through } \\
\text { assessment, communication, } \\
\text { and care planning }\end{array}$ & $\begin{array}{l}\text { Qualitative structured } \\
\text { interview. } N=265\end{array}$ & $\begin{array}{l}\text { Elements of support } \\
\text { and satisfaction }\end{array}$ & $\begin{array}{l}\text { Those who had service contact } \\
\text { more likely to have AIDS, advice } \\
\text { and support main services } \\
\text { provided by staff. Nursing care } \\
\text { described as very good/ } \\
\text { excellent by } 86 \%, 60 \% \text { report } \\
\text { receiving much reassurance and } \\
\text { support }\end{array}$ \\
\hline $\begin{array}{l}\text { WHO } 1992^{80} / \\
\text { qualitative }\end{array}$ & $\begin{array}{l}6 \text { different home } \\
\text { care programmes, } \\
\text { Uganda and } \\
\text { Zambia/HIV/AIDS }\end{array}$ & $\begin{array}{l}\text { (1) Home based patient } \\
\text { and family care linked to } \\
\text { inpatient and outpatient } \\
\text { AIDS unit: medical nursing } \\
\text { psychological and pastoral } \\
\text { care, terminal care. (2) } \\
\text { Home care team working } \\
\text { through hospital and } \\
\text { community clinics: } \\
\text { medication, counselling and } \\
\text { information, terminal care. } \\
\text { Volunteers also provide care. } \\
\text { (3) and (4) Home patient and } \\
\text { family care: counselling, } \\
\text { information, clinical care. } \\
\text { Medical and herbal symptom } \\
\text { control. (5) Terminal } \\
\text { emphasis, provides } \\
\text { continuity of care, reduce } \\
\text { inpatient bed pressure. } \\
\text { Linked to inpatient and } \\
\text { outpatient services. Nursing } \\
\text { and pastoral care. (6) Relief } \\
\text { of inpatient bed pressure, } \\
\text { care for those unable to } \\
\text { attend hospital, linked to } \\
\text { inpatient, outpatient and } \\
\text { pastoral care }\end{array}$ & $\begin{array}{l}\text { Qualitative: staff ( } n=56) \text {, } \\
\text { patient/carer focus }(n=16) \text {, } \\
\text { direct observation }(n=64) \text {, } \\
\text { methods not stated } \\
\end{array}$ & "Effectiveness" & $\begin{array}{l}\text { "Home care improved quality of } \\
\text { life; home care is equivalent or } \\
\text { better than hospital care" no } \\
\text { supporting evidence provided }\end{array}$ \\
\hline $\begin{array}{l}\text { Bunch } 1998^{35} / \\
\text { qualitative }\end{array}$ & $\begin{array}{l}\text { Home hospital, } \\
\text { Oslo, Norway/ } \\
\text { HIV/AIDS }\end{array}$ & $\begin{array}{l}\text { Home nursing care for } \\
\text { hospitalised patients. } \\
\text { Nursing care plans } \\
\text { devised }\end{array}$ & $\begin{array}{l}\text { Post hoc qualitative, } \\
\text { thematic analysis. } N=5 / 64\end{array}$ & Patient satisfaction & $\begin{array}{l}\text { Hospital and other agency link } \\
\text { valued, independence and } \\
\text { feeling physically less unwell. } \\
\text { Disadvantages include anxiety, } \\
\text { fear, and mastering medical } \\
\text { equipment }\end{array}$ \\
\hline $\begin{array}{l}\text { Uys } 2001^{32} \text { and } \\
2002^{33} / \\
\text { qualitative }\end{array}$ & $\begin{array}{l}\text { Integrated home } \\
\text { based community } \\
\text { care, under served } \\
\text { areas. South Africa/ } \\
\text { HIV/AIDS, including } \\
\text { asymptomatic }\end{array}$ & $\begin{array}{l}\text { Links community } \\
\text { caregivers, patients and } \\
\text { families, hospices, clinics } \\
\text { and hospitals. Community } \\
\text { caregivers provide } \\
\text { hygiene care, wound } \\
\text { care, symptom control, } \\
\text { counselling, psychosocial } \\
\text { support, welfare }\end{array}$ & $\begin{array}{l}\text { Retrospective utilisation } \\
\text { focused evaluation, focus } \\
\text { groups. } N=3 / 36 \text { participants } \\
\text { were patients. Methods not } \\
\text { reported }\end{array}$ & $\begin{array}{l}\text { Satisfaction with, } \\
\text { and impact of, } \\
\text { model on patients }\end{array}$ & $\begin{array}{l}\text { Patients valued information } \\
\text { giving, support, welfare } \\
\text { assistance, specialist referrals } \\
\text { and access to care, better } \\
\text { management of Ols. }\end{array}$ \\
\hline As above & & & $\begin{array}{l}\text { Qualitative patient data } \\
\text { reported but methods and } \\
N \text { not stated. }\end{array}$ & Not stated & $\begin{array}{l}\text { Patients reported enhanced } \\
\text { human dignity }\end{array}$ \\
\hline $\begin{array}{l}\text { Moons } 1994^{34} / \\
\text { qualitative }\end{array}$ & $\begin{array}{l}\text { Home care, } \\
\text { Rotterdam, } \\
\text { Netherlands/AIDS, } \\
\text { hospital outpatients: } \\
\text { CDC classification IV } \\
\text { B, C, or D, } \\
\text { Karnofsky = } 60 \text { or } \\
\text { less. Also permanent } \\
\text { address, telephone, } \\
\text { bathroom an } \\
\text { informal caregiver } \\
\text { and GP cooperation }\end{array}$ & $\begin{array}{l}\text { Aim to: improve QoL by } \\
\text { reducing hospital visits. } \\
\text { Home care coordinated } \\
\text { following joint hospital } \\
\text { and community nurse } \\
\text { assessment. Providing } \\
\text { technical, medical and } \\
\text { nursing care, health } \\
\text { education, and psychosocial } \\
\text { support to patients and } \\
\text { caregivers }\end{array}$ & $\begin{array}{l}\text { Qualitative interviews with } \\
\text { patients during receipt of care. } \\
\text { Methods not reported. } \\
\mathrm{N}=9 / 13\end{array}$ & $\begin{array}{l}\text { Patient experience } \\
\text { of the service }\end{array}$ & $\begin{array}{l}\text { Patients valued being able to } \\
\text { avoid hospital visits and remain } \\
\text { at home; less disturbance of } \\
\text { their daily routine; emotional } \\
\text { support received; high quality of } \\
\text { care. However, lack of evening } \\
\text { and weekend access to the } \\
\text { service was a problem. Poor } \\
\text { communication with GP }\end{array}$ \\
\hline
\end{tabular}




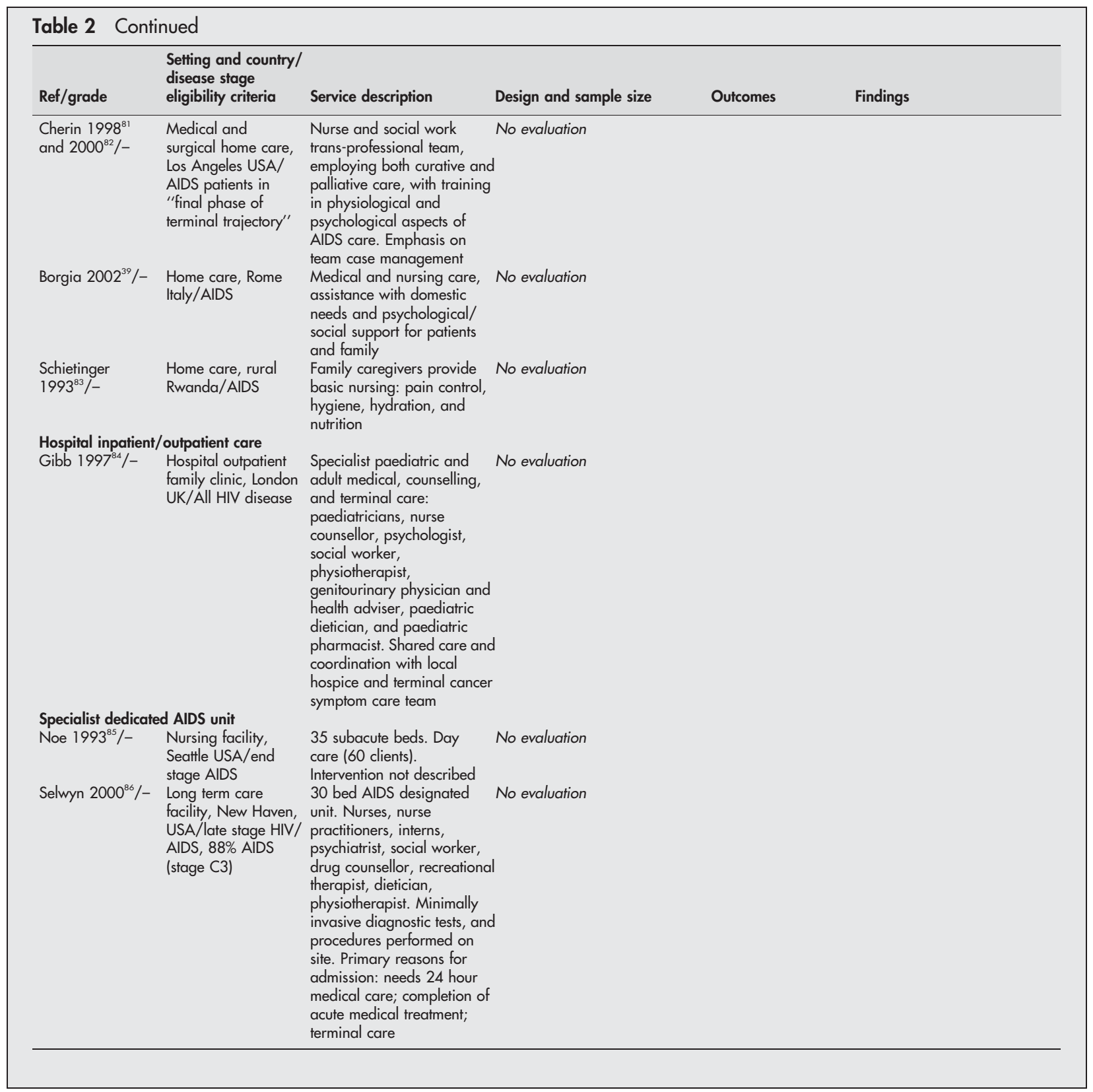

owing to the small sample size and group size imbalance, statistical analysis was not possible.

\section{Grade $3(n=7)$ : weaker evidence}

The following studies used single group longitudinal methods. In a home AIDS palliative team, care was rated as good/ excellent by $100 \%$ of respondents, and qualitative comments reported better communication with palliative care staff than with staff outside the team, and identified helpful elements of care as talking and support. ${ }^{24}$ Among patients receiving home hospice/palliative specialist AIDS care, statistically significant improvements over time were identified in the following domains: symptom control, from $6 \%$ to $33 \%$ reporting no problem from entry to last week of care, $\mathrm{p}=0.00009),{ }^{25}$ and from $4 \%$ to $16 \% \mathrm{p}=0.05$ for the first 2 weeks and from $4 \%$ to $24 \% \mathrm{p}=0.01$ from referral to the point of death ${ }^{26}$ and significant (magnitude unreported) improvements for inpatients of a generic hospice ${ }^{27}$; pain control (from $51 \%$ to $66 \%$ after 2 weeks reporting no problem, $p=0.01$, and from $51 \%$ to $66 \%$ from referral to death $\mathrm{p}=0.0001)^{26}$; family insight from entry to death from $33 \%$ to $67 \%$ reporting as no problem $\mathrm{p}=0.0006)^{25}$; patient anxiety (from $7 \%$ to $16 \%$ from referral to 2 weeks reporting no problem $\mathrm{p}=0.05$, and from $7 \%$ to $47 \%$ from referral to death $\mathrm{p}=0.00005)^{26}$; patient insight $\mathrm{p}=0.04$ (magnitude of change not reported) and spiritual wellbeing $p=0.005$ (magnitude of change not reported). ${ }^{28}$ Conversely, increased pain and discomfort were reported during attendance from 2 weeks to the final 48 hours for patients of another home AIDS hospice team (from $54 \%$ to $68 \%$ ). ${ }^{29}$ Although reductions in pain (from severe pain for $31 \%$ at admission to $3 \%$ in last days) and significant improvement for symptom control (unquantified) were found among patients of an inpatient generic hospice providing AIDS care, no improvement was found for most severe symptoms reported at admission, ${ }^{27}$ and for a community palliative care team, symptom control worsened from referral to death, with the proportion of those reporting severe symptoms rising from $22 \%$ to $46 \%$ $(\mathrm{p}=0.04){ }^{28}$ The study reporting significant symptom improvements from referral to end of life also found 33\% of 
patients having severe symptoms in the last week before death. ${ }^{26}$ In a further study, specialist AIDS palliative pharmacy input was found to improve complex drug management and patient care (although the effect was not quantified)..$^{30}$

\section{Grade $4(n=1)$ : weak evidence}

Using a Likert rating scale of $1=$ excellent to $5=$ poor, a cross sectional evaluation of 52 patients accessing HIV home care found high patient satisfaction with care (mean score 1.6) and personal control over care (mean score 2.2), and with both communication $(84.6 \%$ reporting a professional available to talk) and regularity of contact with staff $(80.6 \%$ satisfied), though perceived health status remained unchanged. ${ }^{31}$

\section{Qualitative $(\mathrm{n}=6)$}

Qualitative data from patients receiving home based care reported patients particularly valuing information giving, support, welfare assistance, and specialist clinical intervention and referrals (focus group data, number unspecified) ) $^{32}$ as well as enhanced human dignity ${ }^{33}$ (patient interviews, number unspecified). Patient interviews in other studies reported less disturbance of daily routine ${ }^{34}$ (nine interviews) ${ }^{35}$ (five interviews) fewer hospital visits and high satisfaction and quality of care $^{34}$ (nine interviews) ${ }^{36}$ (265 interviews). However, quality and satisfaction with care were tempered by the anxiety and fear induced by being cared for at home for a small unspecified number of patients ${ }^{35}$ ( of $n=5$ interviewed patients) and poor weekend/out of hours and primary care communication $^{34}$ (of nine interviews).

\section{DISCUSSION}

In this systematic review of 17 studies, we found that most of the evidence supporting the benefits of palliative care for HIV infected patients is based on seven studies categorised as offering grade 3 evidence. These largely demonstrated that home palliative care and inpatient hospice care significantly improved patient outcomes in the domains of pain and symptom control, anxiety, insight and spiritual wellbeing. However, the need for improvements in pain and symptom management was identified even among specialist services.

Only one study employed the most rigorous evaluation methodology of an RCT (that is, grade 1), and although this study suggested that patient outcomes might be improved in terms of quality of wellbeing and survival, this did not reach statistical significance. The lack of grade 1 evaluations is unsurprising given the extensive literature documenting the challenges and logistical problems with palliative care trials. $^{37}{ }^{38}$ The present review identified a recent RCT of AIDS homecare that failed to adequately recruit and retain patients. ${ }^{39}$ Similar methodological challenges were present in a recent review of palliative and hospice care teams' effect on cancer patient outcomes which found only studies of mainly grade 2 or 3 quality. ${ }^{40}$ The accompanying meta-analysis documented benefits in the domains of pain, symptom control, and trends towards satisfaction and therapeutic intervention, with evidence generally strongest for home care. The qualitative studies identified in this current review were of generally poor methodology, with sample sizes, data collection, and analysis methods seldom reported.

There are several important limitations and caveats to the interpretation of the findings from this systematic review. The lack of (quasi) experimental evaluations means that firm conclusions cannot be drawn regarding the comparative benefit of palliative care. A number of evaluations also did not adequately identify services as palliative although they aimed to provide services such as home based, specialist, and/ or terminal care. Optimal terminal HIV/AIDS care requires a primary focus on palliation, and palliation requires clear and precise terminology to avoid the inconsistency found in clinical instruction for HIV/AIDS terminal care. ${ }^{41}$ The heterogeneous clinical studies included in this review reflect the diverse settings, clinical management strategies, and disease stages relevant to palliative care in HIV disease. A lack of detailed service description for many of the studies meant it was not possible to identify the effective elements of the service that might be replicated, although exceptions were found. For example, the study by Selwyn et al clearly defined the range of patient clinical needs at baseline that were related to the goals of palliative care, and subsequently reported on their full or partial resolution. ${ }^{42} \mathrm{~A}$ further limitation in evaluating palliative care is the set of methodological challenges. These include small sample sizes and attrition, ${ }^{38}$ selection bias recruitment and gate-keeping particularly among those with advanced disease, ${ }^{43}$ aggregation of data from heterogeneous populations, the selection of appropriate outcomes in the measurement of broad psychosocial as well as clinical outcomes, ${ }^{44}$ and the resulting lack of successful RCTs. ${ }^{45}$ In the absence of successful RCTs in areas where compassionate but effective care is needed, overreliance on RCTs may not provide the evidence required. ${ }^{46}$ Other methods such as quasi-experimental and longitudinal cohort designs may be more appropriate for robust but feasible protocols.

There is doubtless greater activity in HIV/AIDS patient management in the field of specialist palliative care than was identified by this review, and the tendency for publication of studies with positive findings may mean an under-representation of studies reporting null findings. We attempted to minimise the bias resulting from terminology by including a broad range of common terms in the search strategy-for example, palliative care approaches being termed comfort care. ${ }^{41}$ This review also was guided by the findings from a recent evaluation of systematic reviews of palliative care services. ${ }^{47}$ Similarly, the grading criteria selected were purposefully broad and inclusive, allowing a full body of evidence rather than being narrow (and RCT focused).

In addition, it may be that current activity in less developed regions is not fully represented, and to address this, end of life HIV care in sub-Saharan Africa has been independently surveyed. ${ }^{48}$ The identification of evidence is also hampered by studies combining diagnosis groups. For example, one study ${ }^{49}$ reported palliative care consultations in an acute care hospital where only $8.3 \%$ of patients had HIV disease, a second study of the benefits of symptom control by a hospital based palliative care consultant and pharmacist also had only $8 \%$ of patients with $\mathrm{HIV}^{50}$ and a third study of parenteral antibiotics in an inpatient palliative care unit reported 15\% of patients having an underlying HIV diagnosis. ${ }^{51}$

The potential for a selection bias in HIV palliative care evaluation was demonstrated in a study that found patients reported lower levels of pain, better insight, less anxiety, and fewer spiritual problems than in providers' assessments. ${ }^{24}$ Patient self reports are also subject to bias because of more unwell patients being unable or unwilling to participate. ${ }^{23}$ There is currently a modest amount of clinical research into HIV palliative care, and future efforts should include both qualitative and quantitative approaches to inform feasibility, acceptability, processes, and preferences as well as outcomes. In addition, well standardised quantitative palliative specific outcome measures such as the Support Team Assessment Schedule (STAS) $)^{21}$ and the Palliative Outcome Scale (POS) ${ }^{52}$ should be considered, as well as a range of quality of care ${ }^{53}$ and quality of life measures ${ }^{54}$ in HIV management.

Our findings have several implications for clinical practice. A review of HIV care found that service delivery models are changing: home hospital care is provided for proportionately 


\section{Key messages}

- The clinical evidence demonstrates that patients with HIV infection require palliative care throughout the disease trajectory in order to control pain and symptoms, to promote adherence through reduction of side effects and toxicity associated with antiretroviral therapy, to manage life limiting co-morbidities such as cancers and end stage liver disease, and to provide quality end of life care for those whom antiretroviral therapy fails or who are unable to access it

- This systematic review of patient outcomes found home palliative care and inpatient hospice care to significantly improve patient outcomes in the domains of pain and symptom control, anxiety, insight, and spiritual wellbeing

- Quality HIV clinical care should offer palliation as a flexible, integrated approach when needed from diagnosis to the end of life across the range of institutional and home care settings alongside new therapeutics

fewer patients, and more frequently for those with less advanced disease. ${ }^{55}$ Palliative care should be part of this shift towards earlier intervention, particularly as our review found palliation to be provided towards advanced and terminal disease stages. Most of the studies were based on patients with end stage disease, and few studies have explored the benefits of palliative care through the disease trajectory. Such longitudinal studies could usefully evaluate the benefits of integrated palliative care, and should be a priority for future research.

Service objectives and the evaluation evidence would benefit from an explicit inclusion of palliative care in service aims and multiprofessional mix. ${ }^{56}$ The historical distinction between early/late stage disease that placed palliative care as solely terminal is inappropriate in the light of the clinical evidence that patients need pain and symptom management (and psychosocial care) throughout the disease trajectory. ${ }^{56}$

Nursing homes, ${ }^{57}$ brain impairment units, $^{59}$ hospices, ${ }^{60}$ hospital palliative care teams, ${ }^{6162}$ prison hospice, ${ }^{63}$ and hospital paediatric wards ${ }^{41}$ face challenges of providing both curative and palliative care for people with HIV/AIDS. Therefore, clinical need throughout the disease trajectory and the range of settings in which patient care is delivered speaks of the need for specialist education and the inclusion of palliative care expertise into the multiprofessional care team. HIV/AIDS palliative care may also be seen as a specialism within palliative care; provider data from a generic hospice highlighted staff concern that the low number of HIV/AIDS referrals prevented them from maintaining skills, fearing poor care as a likely outcome. ${ }^{64}$

Further challenges are presented by resource poor countries and some poorer communities in wealthier nations where the epidemic is most pressing. Psychosocial/"total" care is likely to include additional issues such as orphan care and food security, and care programmes need to consider coverage and not simply strive to provide high quality service to a few patients. ${ }^{65}$ Palliative care remains a novel concept in less developed regions. It is largely absent from the clinical training syllabus for nurses and doctors, and restricted availability of opioids necessitate strategic approaches to ensure the expansion of palliative care. ${ }^{66}$

Patients with HIV/AIDS report needing emotional support, communication, and pain control from their physicians, ${ }^{67}$ and those patients with advanced AIDS describe a good death as having pain/symptom control, optimum quality of life, choosing where to die, having control of their treatment, and having their spiritual needs addressed. ${ }^{68}$ Palliative care remains an essential component of clinical care, embracing pain and symptom management, advance care planning, and psychosocial support. The changing epidemiology of HIV, and the widely divergent management of patients in the developed world compared to those in developing countries, present clear and necessary roles for the integration of palliative approaches to patient management throughout the disease trajectory. The integration of palliative care throughout all stages of patient care, incorporating flexibility and specialist advice needed, shows the curative versus palliative approach to be false. ${ }^{69} 70$ The need for palliative care continues alongside new therapeutics and should not be associated solely with terminal care.

\section{CONTRIBUTORS}

This study was conceived by IJH, VR, and KM; the study protocol was developed by $\mathrm{RH}$ and reviewed and agreed by all authors; RH searched the databases, all authors reviewed the search outputs, and data graded by RH and DK; the manuscript was drafted by RH and $\mathrm{PE}$, and reviewed and commented on by all authors.

\section{Authors' affiliations}

R Harding, I J Higginson, Department of Palliative Care and Policy, Guy's King's and St Thomas's School of Medicine, King's College, London, UK

D Karus, V H Raveis, Center for the Psychosocial Study of Health and Illness, Mailman School of Public Health, Columbia University

P Easterbrook, Academic Department of HIV and GU Medicine, Guy's King's and St Thomas's School of Medicine, King's College, London, UK K Marconi, HIV/AIDS Bureau, Health Resources and Services

Administration, US Department of Health and Human Services

\section{REFERENCES}

1 World Health Organization. National cancer control programmes: policies and managerial guidelines. Geneva: WHO, 2002.

2 WHO/UNAIDS. WHO/UNAIDS global HIV/AIDS statistics 2002. www.unaids.org, 2003.

3 Selwyn PA, Rivard M. Palliative care for AIDS: challenges and opportunities in the era of highly active anti-retroviral therapy. Innovations in End-of-Life Care 2003;4(3)

4 Fantoni M, Ricci F, Del Borgo C, et al. Symptom profile in terminally ill AIDS patients. AIDS Patient Care and Stds 1996;10:171-3.

5 Breitbart W, McDonald MV, Rosenfeld B, et al. Pain in ambulatory AIDS patients. I: Pain characteristics and medical correlates. Pain 1996;68:315-21.

6 Larve F, Fontaine A, Colleau SM. Underestimation and undertreatment of pain in HIV disease: multicentre study. BMJl 1997;314:23-8.

7 Sabin CA. The changing clinical epidemiology of AIDS in the highly active antiretroviral therapy era. AIDS 2002;16(Suppl 4):S61-8.

8 Tamalet C, Fantini J, Tourres C, et al. Resistance of HIV-1 to multiple antiretroviral drugs in France: a 6-year survey (1997-2002) based on an analysis of over 7000 genotypes. AIDS 2003;17:2383-8.

9 Dragsted UB, Gerstoft J, Pedersen C, et al. Randomized trial to evaluate indinavir/ritonavir versus saquinavir/ritonavir in human immunodeficiency virus type 1-infected patients: the MaxCmin 1 Trial. J Infect Dis 2003;188:635-42.

10 Heath KV, Montaner JS, Bondy G, et al. Emerging drug toxicities of highly active antiretroviral therapy for human immunodeficiency virus (HIV) infection. Curr Drug Targets 2003;4:13-22.

11 Mary-Krause M, Cotte L, Simon A, et al. Increased risk of myocardial infarction with duration of protease inhibitor therapy in HIV-infected men AIDS 2003; 17:2479-2486.

12 Rabinstein AA. Stroke in HIV-infected patients: a clinical perspective. Cerebrovascular Diseases 2003;15:37-44.

13 Sperber K, Shao L. Neurologic consequences of HIV infection in the era of HAART. AIDS Patient Care and STDs 2003;17:509-18.

14 Hicks C, Currier J, Sax P, et al. Current managemnt challenges in HIV: tolerability of antiretrovirals amd metabolic complications. AIDS Patient Care and STDs 2003;17:221-33.

15 Matheny SC. Clinical dilemmas in palliative care for HIV infection. J Roy Soc Med 2001;94:449-51.

16 Yeguez JF, Martinez SA, Sands DR, et al. Colorectal malignancies in HIVpositive patients. Am Surg 2003;69:981-7.

17 Powles T, Nelson M, Bower M. HIV-related lung cancer-a growing concern? Int J STD AIDS 2003; 14:647-51. 
18 Easterbrook P, Meadway J. The changing epidemiology of HIV infection: new challenges for HIV palliative care. J Roy Soc Med 2001;94:442-8.

19 Pettricrew $M$. Why certain systematic reviews reach uncertain conclusions. BMJ 2003;326:756-8

20 Clinical Guidance Outcomes Group. Guidance on commissioning cancer services - the research evidence. Leeds: NHS Executive, 1996.

21 Higginson IJ, McCarthy M. Validity of the support team assessment schedule: do staffs' ratings reflect those made by patients or their families? Palliative Medicine 1993:7:219-28.

22 Nickel JT, Salsberry PJ, Caswell RJ, et al. Quality of life in nurse case management of persons with AIDS receiving home care. Research in Nursing and Health 1996;19:91-9.

23 Vincent I, $D^{\prime}$ Herouville $D$, Moulin $P$, et al. Modalities of palliative care in hospitalized patients with advanced AIDS. AIDS Care 2000;12:211-19.

24 Butters E, Higginson I, George R, et al. Palliative care for people with HIV/ AIDS: views of patients, carers and providers. AIDS Care 1993;5:105-16.

25 Koffman J, Higginson I, Naysmith A. Hospice at home-a new service for patients with advanced HIV/AIDS: a pilot evaluation of referrals and outcomes. [see comments.]. Br J Gen Pract 1996:46:539-40.

26 Butters E, Higginson I, George R, et al. Assessing the symptoms, anxiety and practical needs of HIV/AIDS patients receiving palliative care. Quality of Life Res 1992;1:47-51.

27 Gibbs LME, Ellershaw JE, Williams MD. Caring for patients with HIV disease: the experience of a generic hospice. AIDS Care 1997:9:601-7.

28 Butters E, Higginson I. Two HIV/AIDS community support teams: patient characteristics, problems at referral and during the last 6 weeks of life. AIDS Care 1995;7:593-603.

29 Kimball LR, McCormick WC. The pharmacologic management of pain and discomfort in persons with AIDS near the end of life: use of opioid analgesia in the hospice setting. J Pain Symptom Management 1996;11:88-94.

30 Lucas C, Glare PA, Sykes JV. Contribution of a liaison clinical pharmacist to an inpatient palliative care unit. Palliative Medicine 1997;11:209-16

31 Foley ME, Fahs MC, Eisenhandler J, et al. Satisfaction with home healthcare services for clients with HIV: preliminary findings. J Assoc Nurses in AIDS Care 1995;6:20-5

32 Uys LR. Evaluation of the integrated community-based home care model. Curationis S Afr J Nursing 2001;24:75-82.

33 Uys LR. The practice of community caregivers in a home-based HIV/AIDS project in South Africa. J Clin Nursing 2002;11:99-108.

34 Moons M, Kerkstra A, Biewenga T. Specialized home care for patients with AIDS: an experiment in Rotterdam, The Netherlands. J Advanced Nursing 1994:19:1132-40.

35 Bunch EH. AIDS in Norway: a post hoc evaluation of an AIDS home care project. J Clin Nursing 1998; 7:183-7.

36 McCann K. The work of a specialist AIDS home support team: the views and experiences of patients using the service. J Advanced Nursing $1991 ; 16: 832-6$.

37 Rinck GC, Geertrudis AM, van den Bos JK, et al. Methodologic issues in effectiveness research on palliative cancer care: a systematic review. I Clin Oncol 1997; 15:1697-707.

38 McWhinney IR, Bass MJ, Donner A. Evaluation of a palliative care service: problems and piffalls. BMJ 1994;309:1340-2.

39 Borgia P, Schifano P, Spadea T, et al. Assessing effectiveness of home care for persons with AIDS: analysis of methodological problems. AIDS Care 2002;14:645-50.

40 Higginson IJ, Finlay IG, Goodwin DM, et al. Is there evidence that palliative care teams alter end-of-life experiences of patients and their caregivers? J Pain Symptom Management 2003;25:150-68.

41 Henley LD. End of life care in HIV-infected children who died in hospital. Developing World Bioethics 2002;2:39-54.

42 Selwyn PA, Rivard M, Kappell D, et al. Palliative care for AIDS at a large urban teaching hospital: program description and preliminary outcomes. Innovations in End-of-Life Care 2002;4(3).

43 Patrick DL, Curtis JR, Egelberg RA, et al. Measuring and improving the quality of dying and death. Ann Intern Med 2003;139:410-15.

44 Grande GE, Todd CJ. Why are trials in palliative care so difficult? Palliative Medicine 2000;14:69-74.

45 Rinck GC, van den Bos GAM, Kleijnen J, et al. Methodologic issues in effectiveness research on palliative cancer care: a systematic review. J Clin Oncol 1997:14:1697-707.

46 Keeley D. Rigorous assessment of palliative care revisited. BMJ 2004;319:1447-8

47 Goodwin DM, Higginson IJ, Edwards AGK, et al. An evaluation of systematic reviews of palliative care services. J Palliative Care 2002;18:77-83.

48 Harding R, Stewart K, Marconi K, et al. Current HIV/AIDS end of life care in sub-Saharan Africa: a survey of models, services, challenges and priorities. BioMed Central Public Health 2003;3(33)

49 Manfredi PL, Morrison RS, Morris J, et al. Palliative care consultations: how do they impact the care of hospitalized patients? J Pain Symptom Management 2000;20:166-73.

50 McQuillan R, Finlay I, Roberts D, et al. The provision of a palliative care service in a teaching hospital and subsequent evaluation of that service. Palliative Medicine 1996;10:231-9.
51 Clayton J, Fardell B, Hutton-Potts J, et al. Parenteral antibiotics in a palliative care unit: prospective analysis of current practice. Palliative Medicine 2003;17:44-8.

52 Hearn J, Higginson IJ. Development and validation of a core outcome measure for palliative care: the palliative care outcome scale. Quality in Health Care 1999;8:219-27.

53 Wu A, Gifford AL, Asch SM, et al. Quality-of-care indicators for HIV/AIDS Disability Management Health Outcomes 2000:7:315-30.

54 Lin MK, Wu AW, Revicki DA. Incorporating quality of life measures in HIV clinical trials. [Review] [68 refs]. HIV Clinical Trials 2002;3:202-18.

55 Orchi N, Girardi E, Antonucci G, et al. Changing patterns of "hospital-athome" care use by persons with AIDS in the era of combination antiretroviral therapy. AIDS Patient Care and STDs 2001;15:625-32.

56 Armes PJ, Higginson IJ. What constitutes high-quality HIV/AIDS palliative care? J Palliative Care 1999;15:5-12.

57 Heath JM. Care of persons with AIDS in the nursing home. Family Medicine 1998;30:436-40.

58 Buchanan RJ, Wang S, Huang C. Profiles of nursing home residents with HIV. $J$ Health Care for the Poor and Underserved 2002;13:379-91.

59 Baker A. Dedicated palliative care. Nursing Times 1997;93:36-7.

60 Stephenson J, Woods S, Scott B, et al. Clinical update. HIV-related brain impairment: from palliative care to rehabilitation. Int $J$ Palliative Nursing 2000;6:6-11.

61 Frank RR, Campbell ML. Caring for terminally ill patients: one hospital's team approach. J Critical Illness 1999:14:51-5.

62 Adelstein W, Burton S. Palliative care in the acute hospital setting. J Neurosci Nursing 1998;30:200-4.

63 Maull FW. Issues in prison hospice: toward a model for the delivery of hospice care in a correctional setting. Hospice Journal-Physical, Psychosocial, and Pastoral Care of the Dying 1998;13:57-82.

64 Salt S, Wilson L, Edwards A. The use of specialist palliative care services by patients with human immunodeficiency virus-related illness in the Yorkshire Deanery of the northern and Yorkshire region. Palliative Medicine 1998;12:152-60.

65 Drew RS, Mgombane G, Nyaruwa T, et al. Estimating coverage of a community-based home care programme. Tropical Doctor 1997;27:210-14.

66 Barnard D, Jagwe JGM. The introduction of palliative care in Uganda. J Palliative Medicine 2002:5:159-63.

67 Curtis JR, Wenrich MD, Carline JD, et al. Patients' perspectives on physician skill in end-of-life care: differences between patients with COPD, cancer, and AIDS. Chest 2002;122:356-62.

68 Pierson CM, Curtis JR, Patrick DL. A good death: a qualitative study of patients with advanced AIDS. AIDS Care 2002;14:587-98.

69 Selwyn PA, Forstein M. Overcoming the false dichotomy of curative vs palliative care for late stage HIV/AIDS. JAMA 2003;290:806-14

70 Finlay I. UK strategies for palliative care. J Roy Soc Med 2001:94:437-41.

71 Tramarin A, Milocchi F, Tolley K, et al. An economic evaluation of home-care assistance for AIDS patients: a pilot study in a town in northern Italy. AIDS 2004;6:1377-83

72 Crowe S. Home truths. Nursing Times 2001;97:26-7.

73 Martin JP. Hospice and home care for persons with AIDS/ARC: meeting the challenges and ensuring quality. Death Studies 1988;12:463-80.

74 Martin JP. The AIDS home care and hospice program. A multidisciplinary approach to caring for persons with AIDS. Am J Hospice Care 1986;3:35-7.

75 Murie R. A milestone in AIDS care... benefits of a specialist hospice in Edinburgh. Nursing Times 1992;88:24-7.

76 Ley DC. Caring for persons with AIDS: initiatives. The Casey House model. J Palliative Care 1988:4:111-15.

77 Moss V. Caring for persons with AIDS: initiatives. The Mildmay approach. J Palliative Care 1988;4:102-6.

78 Cumming M. Difficulties in developing a respite program. J Palliative Care 1993;9:18-22.

79 Singh S, Hawkins D, Connolly M, et al. Model of care for the hospital treatment of individuals with HIV infection. Health Trends 1991;23:55-9.

80 WHO Global Programme on AIDS. Battling AIDS through home care in Uganda and Zambia. Caring 1992;11:56-70.

81 Cherin DA, Huba GJ, Brief DE, et al. Evaluation of the transprofessional model of home health care for HIV/AIDS. Home Health Care Services Quarterly 1998;17:55-72.

82 Cherin DA, Melchior LA, Brief DE. Issues in implementing and evaluating a managed care home health care system for HIV/AIDS: visiting nurse association foundation of Los Angeles. Drugs and Society 2000;16:203-22.

83 Schietinger H, Almedal C, Marianne BN, et al. Teaching Rwandan families to care for people with AIDS at home. Hospice Journal-Physical, Psychosocial, and Pastoral Care of the Dying 1993;9:33-53

84 Gibb DM, Masters J, Shingadia D, et al. A family clinic-optimising care for HIV-infected children and their families. Arch Dis Childh 1997;77:478-82.

85 Noe M. The Bailey-Boushay House: an AIDS facility. J Ahima 1993;64:46-7.

86 Selwyn PA, Goulet JL, Molde S, et al. HIV as a chronic disease: implications for long-term care at an AIDS-dedicated skilled nursing facility. J Urban Health 2000;77:187-203. 